Pacific

Journal of

Mathematics

ON THE CUT LOCUS IN ALEXANDROV SPACES AND APPLICATIONS TO CONVEX SURFACES

TUdor ZAMFIRESCU

Volume $217 \quad$ No. 2

December 2004 


\title{
ON THE CUT LOCUS IN ALEXANDROV SPACES AND APPLICATIONS TO CONVEX SURFACES
}

\author{
TUdOR ZAMFIRESCU
}

\begin{abstract}
Alexandrov spaces are a large class of metric spaces that includes Hilbert spaces, Riemannian manifolds and convex surfaces. In the framework of Alexandrov spaces, we examine the ambiguous locus of analysis and the cut locus of differential geometry, proving a general bisecting property, showing how small the ambiguous locus must be, and proving that typically the ambiguous locus and a fortiori the cut locus are dense.
\end{abstract}

\section{Introduction}

A metric space is called an Alexandrov space if it is an intrinsic metric space with curvature bounded below in the sense of Alexandrov (see definition on page 376). Such spaces were introduced by A.D. Alexandrov in 1955, along with spaces with curvature bounded above.

Hilbert spaces, Riemannian manifolds, convex surfaces, and convex subsets of these are examples of Alexandrov spaces.

This paper considers the ambiguous locus of analysis and the cut locus of differential geometry in the framework of Alexandrov spaces. We prove a general bisecting property, then show how small the ambiguous locus must be, and finally prove that typically the ambiguous locus and a fortiori the cut locus must be dense.

In fact, we discover that the apparently distant notions of cut locus and ambiguous locus share a common soul. Some more (metamathematical) searching brings to light another two independent developments of the same notion.

The ambiguous locus has been investigated by - among others - de Blasi and Myjak [3], [4], [5]; de Blasi, Kenderov and Myjak [6]; Myjak and Rudnicki [21]; Zhivkov [41]; de Blasi and Zamfirescu [7].

The cut locus, introduced by Poincaré in 1905 [25], received its name from Whitehead in 1936; it was studied by Myers in 1935-36 [19], [20], and subsequently by many other authors in the context of Riemannian geometry. Their contributions grew to what is today a large, well-established body of results. 
More recently, Otsu and Shioya [22], Shiohama and Tanaka [27], Itoh [15], Zamfirescu [38] and others have investigated the cut locus in Alexandrov spaces. Shiohama and Tanaka considered in [27] the cut locus with respect to a compact set.

Independently, A. Rivière [26] studied the cut locus (under the name of "nervure") with respect to closed subsets of a Euclidean space.

A fourth independent appearance of the cut locus (or, more exactly, of the ambiguous locus), with a more applied background, under the name of "medial axis", appears in papers by Lee [17], Lee and Drysdale [18], Yap [29], Choi, Choi and Moon [10], and others.

Kunze [16] and Zamfirescu [39] have treated problems on the cut locus in the case of closed convex hypersurfaces without any differentiability assumptions.

By making use of the notion of porosity we shall see how small ( $\sigma$-porous) the ambiguous locus must always be, and also how large (dense) it can sometimes be. These results relate to work in Banach spaces and in Riemannian manifolds.

Definitions. We work in a metric space $(\mathcal{A}, \rho)$.

A segment between two distinct points is a shortest path between thema minimal segment in Otsu and Shioya's terminology [22].

A segment between a point $x$ and a closed set $K$ not containing $x$ is an arc between $x$ and a point of $K$, not longer than any other such arc.

A geodesic is a curve which is locally a segment (for a precise definition see, e.g., [38]).

A geodesic triangle is a triangle with segments as sides.

Let $S_{k}$ denote the 2-dimensional complete simply-connected Riemannian manifold of constant curvature $k<0$ (a Lobachevskii plane).

If $a, b, c$ belong to the metric space $(\mathcal{A}, \rho)$, let $\angle^{*} a b c$ denote the angle of the geodesic triangle in $S_{k}$ of side-lengths $\rho(a, b), \rho(b, c), \rho(c, a)$, opposite to the side of length $\rho(c, a)$.

Here, $(\mathcal{A}, \rho)$ is called an intrinsic metric space if any two points admit a midpoint. An Alexandrov space is a complete intrinsic metric space $(\mathcal{A}, \rho)$ such that every point of $\mathcal{A}$ has a neighbourhood in which, for any four distinct points $a, b, c, d$, we have

$$
\angle^{*} b a c+\angle^{*} c a d+\angle^{*} d a b \leq 2 \pi .
$$

This angle condition says that $(\mathcal{A}, \rho)$ has curvature bounded below by $k$ in the sense of A.D. Alexandrov (note the dependence of $\angle^{*}$ on $k$ ).

Berestovskii [2] proved that a complete intrinsic metric space $(\mathcal{A}, \rho)$ is an Alexandrov space if and only if every point of $\mathcal{A}$ has a neighbourhood in which any four points admit an isometric embedding in $S_{k^{\prime}}$ for some $k^{\prime} \geq k$. 
Several other characterizations of Alexandrov spaces are given by Burago, Gromov and Perelman in [9]. We use some basic concepts and results developed in [9] and further investigated by Perelman [23], [24]. So, for example, in any Alexandrov space a geodesic starting at $x$ has a direction at $x$ (see the definition below) and the angle between two geodesics exists. Moreover, geodesics do not branch. The interested reader should consult the recent book of Burago, Burago and Ivanov [8].

\section{Prerequisites}

If $x$ is a point in the Alexandrov space $\mathcal{A}$, the space $\Sigma_{x}$ of directions at $x$ is defined as the completion of the metric space $\Sigma_{x}^{\prime}$ consisting of classes of geodesics starting at $x$, all the geodesics of the same class overlapping, and the distance being the angle between representatives (see [9], p. 23). We shall identify the elements of $\Sigma_{x}^{\prime}$ with the directions of representatives. Every space of directions is known to be itself an Alexandrov space if $\mathcal{A}$ has finite dimension [9].

Let $\overline{x y}$ denote the direction of the segment $x y$ at $x$.

We say that a set $A \in \mathcal{A}$ has a direction $\tau$ at its accumulation point $x$ if, for $y \rightarrow x$ with $y \in A \backslash\{x\}, \overline{x y}$ converges in $\Sigma_{x}$ to $\tau$.

If $p a, p b$ are geodesics, let $\angle a p b$ denote the angle between $p a$ and $p b$, i.e., the distance from $\overline{p a}$ to $\overline{p b}$.

We now recall three basic results from [9].

Lemma 1 ([9], p. 6). If pa, pb, pc are geodesics in an Alexandrov space,

$$
\angle a p b+\angle b p c+\angle c p a \leq 2 \pi .
$$

Here is a generalized form of Toponogov's comparison theorem:

Lemma 2 ([9], p. 7). For any geodesic triangle abc in an Alexandrov space,

$$
\angle^{*} a b c \leq \angle a b c, \quad \angle^{*} b c a \leq \angle b c a, \quad \angle^{*} c a b \leq \angle c a b .
$$

Lemma 3 ([9], p. 6). If in an Alexandrov space the segment $p_{i} q_{i}$ converges to $p q$ and the segment $p_{i} r_{i}$ converges to $p r$, then

$$
\angle q p r \leq \liminf _{i \rightarrow \infty} \angle q_{i} p_{i} r_{i} .
$$

Let $\mathcal{A}$ be an Alexandrov space and $K \subset \mathcal{A}$ a closed set.

The cut locus $C(K)$ of $K$ is the set of all points $x \in \mathcal{A}$ such that there is a segment from $x$ to $K$ not extendable as a segment beyond $x$.

The multijoined locus of $K$ is the set $M(K)$ of all points $x \in \mathcal{A}$ such that the distance from $x$ to $K$ is realized by at least two distinct segments from $x$ to (not necessarily distinct) points in $K$. The points of $M(K)$ are said to be multijoined to $K$ (see also [37]). 
The ambiguous locus of $K$ is the set $A(K)$ of all points $x \in \mathcal{A}$ such that the distance from $x$ to $K$ is realized by at least two segments from $x$ to distinct points in $K$.

If $K$ consists of a single point $x$, we write $C(x)$ and $M(x)$ instead of $C(\{x\})$ and $M(\{x\})$, respectively.

A point in $\mathcal{A}$ is called an endpoint if it is not interior to any segment; let $E$ denote the set of all endpoints of $\mathcal{A}$.

Clearly, $A(K) \subset M(K) \subset C(K)$ and $E \subset C(K)$.

The open ball with centre $x \in \mathcal{A}$ and radius $\alpha$ is denoted by $B(x, \alpha)$.

The boundary of a compact convex set with interior points in $\mathbb{R}^{n}$ is called a convex hypersurface. The space of all convex hypersurfaces, equipped with the Pompeiu-Hausdorff metric, is a Baire space.

Most (or typical) means "all, except those in a set of first Baire category".

Lemma 4 ([31]). On most convex hypersurfaces, most points are endpoints.

\section{Equidistant sets}

Both the ambiguous locus and the cut locus are known to enjoy a bisecting property with respect to certain pairs of segments.

Let $a, b$ be (possibly coinciding) points in $\mathcal{A}$. The set $E(a, b)$ of all points joined by equally long but distinct segments with $a$ and $b$ will be called the equidistant set of $\{a, b\}$. Thus $E(a, b)$ coincides with $A(\{a, b\})$ if $a \neq b$, and with $M(\{a\})$ if $a=b$.

We shall prove a bisecting property of $E(a, b)$ which lies at the core of these phenomena.

Let $x \in E(a, b)$, and consider distinct segments $x a, x b \subset \mathcal{A}$ and $\alpha, \beta \subset \Sigma_{x}$ such that $\alpha=\overline{x a}, \beta=\overline{x b}$. Also, suppose $\alpha \beta$ is a segment in $\Sigma_{x}$.

A bisector of $x a, x b$ is a nondegenerate arc $\Gamma \subset E(a, b)$ starting at $x$ such that, for any $y \in \Gamma \backslash\{x\}$ :

1) There is a segment $x y$ with $\overline{x y} \in \alpha \beta$.

2) There are segments $y a, y b$ such that $y a \rightarrow x a$ and $y b \rightarrow x b$ for $y \rightarrow x$. This name is explained by the next theorem.

There may exist no bisector of $x a, x b$. This is the case, for example, if $a=b$ and $a, x$ are antipodal on the standard sphere, because then $E(a, a)$ is a single point and cannot include any nondegenerate arc. But we may also have no bisector if $a \neq b$ : consider a smooth convex (2-dimensional) surface with precisely two segments from $x$ to $a$, say $\sigma_{1}, \sigma_{2}$, and precisely one segment $\sigma_{3}$ from $x$ to $b$ such that, for $s_{i} \in \operatorname{int} \sigma_{i}$,

$$
\angle s_{1} x s_{2}+\angle s_{2} x s_{3}=\angle s_{1} x s_{3}<\pi .
$$

Then $\sigma_{1}$ and $\sigma_{3}$ admit no bisector, because condition 2 is not satisfied. In this case, however, $\sigma_{1}, \sigma_{2}$, as well as $\sigma_{2}, \sigma_{3}$, would admit a bisector each. 
Theorem 1. Any bisector of $x a, x b$ has a direction at $x$, namely the midpoint of $\alpha \beta$, where $\alpha=\overline{x a}$ and $\beta=\overline{x b}$.

Proof. Let $\Gamma$ be a bisector of $x a, x b$. If $y \in \Gamma \backslash\{x\}$ converges to $x$ then $\angle^{*} x a y \rightarrow 0$, which implies $\angle^{*} a x y+\angle^{*} a y x \rightarrow \pi$. Analogously, $\angle^{*} b x y+$ $\angle^{*}$ by $x \rightarrow \pi$. Hence

$$
\left\llcorner^{*} a x y+\left\llcorner^{*} b x y+\left\llcorner^{*} a y x+\angle^{*} b y x \rightarrow 2 \pi .\right.\right.\right.
$$

By Lemma 2,

$$
\liminf _{y \rightarrow x}(\angle a x y+\angle b x y+\angle a y x+\angle b y x) \geq 2 \pi .
$$

Since $\overline{x y} \in \alpha \beta$,

$$
\angle a x b=\angle a x y+\angle b x y .
$$

Hence

$$
\liminf _{y \rightarrow x}(\angle a x b+\angle a y x+\angle b y x) \geq 2 \pi .
$$

Suppose

$$
\limsup _{y \rightarrow x}(\angle a x b+\angle a y x+\angle b y x)>2 \pi .
$$

By Lemma 3,

$$
\liminf _{y \rightarrow x} \angle a y b \geq \angle a x b .
$$

Then

$$
\limsup _{y \rightarrow x}(\angle a y b+\angle a y x+\angle b y x)>2 \pi,
$$

which contradicts the inequality

$$
\angle a y b+\angle a y x+\angle b y x \leq 2 \pi,
$$

which holds, by Lemma 1, for all $y$. Hence

$$
\angle a x b+\angle a y x+\angle b y x \rightarrow 2 \pi .
$$

Using Lemma 2 again, we get $\angle a x y-\angle^{*} a x y \rightarrow 0, \angle b x y-\angle^{*} b x y \rightarrow 0$, $\angle a y x-\angle^{*} a y x \rightarrow 0, \angle b y x-\angle^{*} b y x \rightarrow 0$. But $\angle^{*} a x y=\angle^{*} b x y$; therefore

$$
\angle a x y-\angle b x y \rightarrow 0
$$

and

$$
\lim _{y \rightarrow x} \angle a x y=\lim _{y \rightarrow x} \angle b x y=\frac{1}{2} \angle a x b .
$$

Thus, the proof is finished. 


\section{Multijoined loci in Alexandrov spaces: the arbitrary case}

A set $A$ in a metric space $(\mathcal{M}, \rho)$ is said to be porous at $x \in \mathcal{M}$ if there is a number $\alpha>0$ such that every ball centered at $x$ contains a ball $B(y, \alpha \rho(x, y))$ disjoint from $A$. It is called porous if it is porous at all its points, and $\sigma$-porous if it is a countable union of porous sets.

The main result of this section establishes the $\sigma$-porosity of the multijoined locus of any closed set in an arbitrary Alexandrov space $\mathcal{A}$. This extends results of Gruber [12], [13] and the author [35].

Otsu and Shioya [22] have shown that the cut locus $C(x)$ of a point $x$ in an $n$-dimensional Alexandrov space $\mathcal{A}$ has $n$-dimensional Hausdorff measure 0 . However we cannot expect $C(x)$ to be $\sigma$-porous because, by Lemma 4 , there are Alexandrov spaces in which $C(x)$ is residual. About the multijoined locus Otsu and Shioya proved the stronger assertion $\operatorname{dim} M(x) \leq n-1$ [22].

Also, Shiohama and Tanaka described in detail, for $n=2$, the structure of $C(K)$ and $M(K)$ for any compact set $K[\mathbf{2 7}]$. According to them, $M(K)$ is a countable union of Jordan arcs.

Unfortunately, the Hausdorff dimension being at most $n-1$ does not imply $\sigma$-porosity (see Zajíček [30]), nor are Jordan arcs necessarily $\sigma$-porous (see Foran $[\mathbf{1 1}])$.

Lemma 5. Let $\mathcal{A}$ be an Alexandrov space and let $A \subset \mathcal{A}$ be such that, for some points $x, x^{\prime}$ in $\mathcal{A}$ and some positive number $\varepsilon$, all points $z$ in a neighbourhood of $x$ that satisfy $\angle^{*} z x x^{\prime} \leq \varepsilon$ do not belong to $A$. Then $A$ is porous at $x$.

Proof. Let $N$ be the neighbourhood of $x$ considered in the statement. It suffices to take $\alpha=\sin \varepsilon$ in the definition of porosity. Then, for any segment $x x^{\prime}$ and any point $y \in x x^{\prime}$,

$$
z \in B(y, \alpha \rho(x, y)) \cap N \Longrightarrow \sin \angle^{*} z x y \leq \alpha \Longrightarrow \angle^{*} z x x^{\prime} \leq \varepsilon .
$$

Hence $z \in N \backslash A$, and $A$ is porous at $x$.

Theorem 2. In an Alexandrov space, the multijoined locus of any closed set is $\sigma$-porous.

Proof. Let $\mathcal{A}$ be an Alexandrov space and let $K \subset \mathcal{A}$ be closed. For any $m \in \mathbb{N}$, let $M_{m} \subset M(K)$ be the set of all points $u$ joined with $K$ by (at least) two segments making an angle at $x$ not less than $m^{-1}$. We have

$$
M(K)=\bigcup_{n=1}^{\infty} M_{m},
$$

and we only have to prove that $M_{m}$ is porous for each $m \in \mathbb{N}$.

Consider $u, v \in M_{m}$ and let $a, b$ be interior points of the two segments from $u$ to $K$ under consideration. If simultaneously

$$
\angle a u v>\pi-(2 m)^{-1} \text { and } \angle b u v>\pi-(2 m)^{-1},
$$


then

$$
\angle a u v+\angle b u v+\angle a u b>2 \pi,
$$

which contradicts Lemma 1. Thus, there exists a segment $u x$ from $u$ to $K$ such that, in the geodesic triangle $x u v$,

$$
\angle x u v \leq \pi-(2 m)^{-1} .
$$

By Lemma $2, L^{*} x u v \leq \pi-(2 m)^{-1}$ too.

Now, keep $v$ fixed and let $u \in M_{m}$ converge to $v$. Then $\angle^{*} u x v \rightarrow 0$. But this yields $L^{*} x v u>(3 m)^{-1}$ for all $u \in M_{m}$ in some neighbourhood of $v$. Then, by Lemma $5, M_{m}$ is porous at $v$. Thus, $M_{m}$ is porous, $M(K)$ is $\sigma$-porous, and the proof is finished.

For the special case of a point $x$ on a convex surface, the $\sigma$-porosity of $M(x)$ was proved in [35] (see also Gruber [12], [13]).

\section{Ambiguous and multijoined loci in Alexandrov spaces: the generic case}

In this section we consider the ambiguous locus in typical cases. We investigate typical compact sets or take the ambient space to be typical. Let $\mathcal{K}(\mathcal{A})$ be the space of all compact sets in the Alexandrov space $(\mathcal{A}, \rho)$, endowed with its usual Pompeiu-Hausdorff metric $h$, based on $\rho$.

The next theorem generalizes Theorem 1 from [34] considerably. This result of [34] has already been repeatedly strengthened, for example in [3], $[4],[5],[6],[41],[7]$.

Theorem 3. For most compact sets in a separable Alexandrov space of dimension at least 2 , the ambiguous locus is dense.

Proof. Let $B\left(x_{0}, \varepsilon\right)$ be an open ball in the Alexandrov space $\mathcal{A}$. We prove that the compact sets $K \subset \mathcal{A}$ for which the ambiguous locus $A(K)$ does not meet $B\left(x_{0}, \varepsilon\right)$ form a nowhere dense set.

Indeed, in any open set $\mathcal{O} \subset \mathcal{K}(\mathcal{A})$, there exists a compact set $K$ avoiding $x_{0}$. Let $y_{0} \in K$ be a point closest to $x_{0}$ and take $y_{1}$ different from $y_{0}$ on a segment $\sigma$ from $x_{0}$ to $y_{0}$, so that $K \cup\left\{y_{1}\right\} \in \mathcal{O}$. There is a whole ball $B\left(y_{1}, \eta\right)$ disjoint from $K$ such that for any finite set $F \subset B\left(y_{1}, \eta\right), K \cup F \in \mathcal{O}$.

Since $\operatorname{dim} \mathcal{A} \geq 2$, we can choose $y_{2} \in B\left(y_{1}, \eta\right) \backslash \sigma$.

Consider the point $y_{3} \in \sigma$ with $\rho\left(x_{0}, y_{2}\right)=\rho\left(x_{0}, y_{3}\right)$. Then $y_{3} \in B\left(y_{1}, \eta\right)$ too. Let $\sigma_{2}, \sigma_{3}$ be segments from $x_{0}$ to $y_{2}, y_{3}$ respectively. Choose points $x_{2} \in \sigma_{2}, x_{3} \in \sigma_{3}$ such that $\rho\left(x, x_{2}\right)=\rho\left(x, x_{3}\right)<\varepsilon$. Clearly, $\rho\left(x_{2}, y_{3}\right)>$ $\rho\left(x_{2}, y_{2}\right), \rho\left(x_{3}, y_{2}\right)>\rho\left(x_{3}, y_{3}\right)$.

Let

$$
\nu<\min \left\{\rho\left(x_{2}, y_{3}\right)-\rho\left(x_{2}, y_{2}\right), \rho\left(x_{3}, y_{2}\right)-\rho\left(x_{3}, y_{3}\right)\right\}
$$


If $h\left(K^{\prime}, K \cup\left\{y_{2}, y_{3}\right\}\right)<\nu / 2$ in $\mathcal{K}(\mathcal{A})$, then $K^{\prime}$ meets both $B\left(y_{1}, \nu / 2\right)$ and $B\left(y_{2}, \nu / 2\right)$. Therefore, for $i=2,3$, the point of $K^{\prime}$ closest to $x_{i}$ lies in $B\left(y_{i}, \nu / 2\right)$. The function

$$
f(x)=d\left(x, K^{\prime} \cap B\left(y_{2}, \nu / 2\right)\right)-d\left(x, K^{\prime} \cap B\left(y_{3}, \nu / 2\right)\right),
$$

where $d(x, M)$ means the infimum of $\rho(x, y)$ for $y \in M$, is continuous, $f\left(x_{2}\right)<0$ and $f\left(x_{3}\right)>0$. Thus there is a point $x \in x_{2} x_{0} \cup x_{0} x_{3}$ with $f(x)=0$; that is, $x \in A\left(K^{\prime}\right)$.

Hence the set $\mathcal{K}_{m, n} \subset \mathcal{K}(\mathcal{A})$ of all compact sets $K$ for which $A(K) \cap$ $B\left(x_{m}, 1 / n\right)=\emptyset$ is nowhere dense. Since $\mathcal{A}$ is separable, $\left\{x_{m}\right\}_{m=1}^{\infty}$ can be chosen to be dense in $\mathcal{A}$, and then the set $\bigcup_{m, n=1}^{\infty} \mathcal{K}_{m, n}$ of all compact sets with nondense ambiguous locus is of first Baire category.

For an interesting analog of Theorem 3 concerning convex hypersurfaces instead of compact sets, see [40].

There are Alexandrov spaces in which the multijoined locus is dense for more compact sets than "just" those of a second Baire family. More precisely, in these spaces, the multijoined locus of any element of an open subset of $\mathcal{K}(\mathcal{A})$ is dense in its complement.

The following result strengthens in several directions Theorem 2 in [35] and C. Vîlcu's second assertion in the first theorem of [28].

Theorem 4. Let the compact Alexandrov space $\mathcal{A}$ be an $n$-dimensional topological manifold ( $n \geq 2$, finite), and $A \subset \mathcal{A}$. Assume that the set of endpoints of $\mathcal{A}$ is dense in $A$ (with respect to its relative topology) and $K$ is a closed subset of a union $B$ of components of $\mathcal{A} \backslash A$. If $B$ is not dense in $\mathcal{A}$ then the multijoined locus $M(K)$ is dense in the interior of $\mathcal{A} \backslash B$.

Proof. Suppose there is an open set $O \subset \mathcal{A} \backslash B$ homeomorphic to $\mathbb{R}^{n}$ and disjoint from $M(K)$. Since every point $y \in \mathcal{A} \backslash M(K)$ is joined by precisely one segment $\sigma_{y}$ to the closest point $\pi_{y}$ of $K$, the mapping $y \mapsto \sigma_{y}$ is continuous in $O$. The mapping $y \mapsto \pi_{y}$ is continuous too.

If $0 \leq r \leq 1$ and $\phi(y, r)$ denotes the point of $\sigma_{y}$ at distance $r \rho\left(y, \pi_{y}\right)$ from $y$, then $\phi$ is continuous in both variables.

Moreover the function $\psi_{r}$ defined by $\psi_{r}(y)=\phi(y, r)$ is injective for $0 \leq$ $r<1$, since geodesics do not branch and therefore $y \neq y^{\prime}$ implies $\sigma_{y} \subset \sigma_{y^{\prime}}$ or $\sigma_{y^{\prime}} \subset \sigma_{y}$ or $\sigma_{y} \cap \sigma_{y^{\prime}}=\left\{\pi_{y}\right\} \cap\left\{\pi_{y^{\prime}}\right\}$. The inverse of $\psi_{r}$, defined on $\psi_{r}(O)$, is obviously continuous too.

Let $y_{0} \in O$. For some $r$ distinct from $1, \psi_{r}\left(y_{0}\right)$ belongs to $A$ and is different from $y_{0}$. We may consider $O$ chosen such that $\psi_{r}(O)$ lies in a neighbourhood of $y_{0}$ homeomorphic to $\mathbb{R}^{n}$. Then $\psi_{r}(O)$ is open (see, e.g., [14, Theorem 6-54]). Hence $\psi_{r}(O) \cap A$ is nonempty and open in the relative topology of $A$. Now choose the endpoint $z$ in this set. Then $z$ must belong to a segment $\sigma_{y}$ with $y \in O$, and is not an endpoint of $\sigma_{y}$. This contradiction completes the proof. 
Corollary 1. In a space $\mathcal{A}$ as in Theorem 4, assume that the closed set $K$ is strictly included in the open set $O$, and that the set of endpoints of $\mathcal{A}$ is dense in $O \backslash K$. Then $M(K)$ is dense in $\mathcal{A} \backslash K$.

Example. Consider a smooth torus $T$ embedded in $\mathbb{R}^{3}$ and a point $p \in T$ of positive Gauss curvature. Near $p$ and outside $T$ take a convex hypersurface $C$ of the type described in Lemma 4 .

If $C$ is small enough and close enough to $p$, each line segment $s$ joining a point of $C$ with a point of $T$ and not meeting the interiors (bounded components of the complements) of $C$ and $T$ has an endpoint on $T$ of positive Gauss curvature. The union of all line segments $s$ has a boundary $S$. Then the (topological) closure of

$$
C \cup S \cup T \backslash((C \cap S) \cup(S \cap T))
$$

is a torus $T^{\prime}$, the endpoints of which lie densely in $C \backslash S$. By Corollary 1, for any compact set $K \subset C$ disjoint from $S, M(K)$ is dense in $T^{\prime} \backslash K$.

Theorem 5. On most convex hypersurfaces $S$, for any compact set $K \subset S$, the multijoined locus $M(K)$ is dense in $S \backslash K$.

This follows from Corollary 1 and Lemma 4.

By Theorems 3 and 5, we can say that every compact set on most convex hypersurfaces and most compact sets on every convex hypersurface have multijoined loci dense in their complements.

In a Baire metric space, "nearly all" means "all, except those in a $\sigma$-porous set" [32].

Lemma 6. On any convex hypersurface, nearly all compact sets are porous.

This follows from Theorem 2 in $[33]$ applied to $\mathbb{R}^{n-1}$, together with the observation that the convex hypersurface can be tiled into finitely many pieces admitting bi-Lipschitz bijections to pieces of $\mathbb{R}^{n-1}$, while porosity and $\sigma$-porosity are invariant under bi-Lipschitz transformations.

Lemma 6 and Theorem 5 immediately imply the following result:

Theorem 6. On most convex hypersurfaces $S$, for nearly all compact sets the multijoined locus is dense on $S$.

Suggestion. Show that Theorem 6 is also true for the ambiguous locus instead of the multijoined locus, although Theorem 5 is not.

Acknowledgement. I am very much indebted to the referee and to Silvio Levy, whose expertise influenced the final form of this paper. 


\section{References}

[1] A.D. Alexandrow, Die innere Geometrie der konvexen Flächen. Akademie-Verlag, Berlin, 1955, MR 0071041 (17,74d), Zbl 0065.15102.

[2] V.N. Berestovskii, Spaces with bounded curvature and distance geometry, Siberian Math. J., 27 (1986), 11-25, MR 0847410 (88e:53110), Zbl 0612.53047.

[3] F. de Blasi and J. Myjak, Ambiguous loci of the nearest point mapping in Banach spaces, Arch. Math., 61 (1993), 377-384, MR 1236316 (94i:41043), Zbl 0822.46012.

[4] F. de Blasi and J. Myjak, On compact connected sets in Banach spaces, Proc. Amer. Math. Soc., 124 (1996), 2331-2336, MR 1363408 (97e:46014), Zbl 0860.46008.

[5] F. de Blasi and J. Myjak, Ambiguous loci in best approximation theory, Approximation theory, spline functions and applications (Maratea, 1991), 341-349, NATO Adv. Sci. Inst. Ser. C Math. Phys. Sci., 356, Kluwer Acad. Publ., Dordrecht, 1992, MR 1165980 (93e:41049), Zbl 0751.41020.

[6] F. de Blasi, P. Kenderov and J. Myjak, Ambiguous loci of the metric projection onto compact starshaped sets in a Banach space, Monatsh. Math., 119 (1995), 23-36, MR 1315681 (96d:52004), Zbl 0818.54026.

[7] F. de Blasi and T. Zamfirescu, Cardinality of the metric projection on typical compact sets in Hilbert spaces, Math. Proc. Cambridge Phil. Soc., 126 (1999), 37-44, MR 1681652 (2000e:41050), Zbl 0923.46022.

[8] D. Burago, Y. Burago and S. Ivanov, A Course in Metric Geometry, Graduate Studies in Mathematics, 33, American Mathematical Society, Providence, RI, 2001, MR 1835418 (2002e:53053), Zbl 0981.51016.

[9] Y. Burago, M. Gromov and G. Perelman, A.D. Alexandrov spaces with curvature bounded below, Russian Math. Surveys, 47 (1992), 1-58, MR 1185284 (93m:53035), Zbl 0802.53018.

[10] H.I. Choi, S.W. Choi and H.P. Moon, Mathematical theory of medial axis transform, Pacific J. Math., 181 (1997), 57-88, MR 1491036 (99m:53008), Zbl 0885.53004.

[11] J. Foran, Continuous functions need not have $\sigma$-porous graphs, Real Anal. Exchange, 11 (1985/86), 194-203, MR 0828490 (87d:26003), Zbl 0607.26005.

[12] P. Gruber, Geodesics on typical convex surfaces, Atti Accad. Naz. Lincei Rend. Cl. Sci. Fis. Mat. Natur. (8), 82 (1988), 651-659, MR 1139812 (93a:53054), Zbl 0741.52006.

[13] P. Gruber, Baire categories in convexity, in 'Handbook of Convex Geometry', Vol. B, North-Holland, Amsterdam, 1993, 1327-1346, MR 1243011 (94i:52003), Zbl 0791.52002.

[14] J.G. Hocking and G.S. Young, Topology, (Second edition), Dover Publications, Inc., New York, 1988, MR 1016814 (90h:54001), Zbl 0718.55001.

[15] J.-I. Itoh, The length of a cut locus on a surface and Ambrose's problem, J. Differential Geom., 43 (1996), 642-651, MR 1412679 (97i:53038), Zbl 0865.53031.

[16] J. Kunze, Der Schnittort auf konvexen Verheftungsflächen, Mathematische Monographien, 9 VEB Deutscher Verlag der Wissenschaften, Berlin, 1969, MR 0271879 (42 \#6760), Zbl 0211.54201.

[17] D.T. Lee, Medial axis transform of a planar shape, IEEE Trans. Pattern Anal. Mach. Intell., 4 (1982), 363-369, Zbl 0483.68085.

[18] D.T. Lee and R.L. Drysdale, Generalization of Voronoi diagrams in the plane, SIAM J. Comput., 10 (1981), 73-87, MR 0605604 (83i:90048), Zbl 0454.68083. 
[19] S.B. Myers, Connections between differential geometry and topology I: Simply connected surfaces, Duke Math. J., 1 (1935), 376-391, Zbl 0012.27502.

[20] S.B. Myers, Connections between differential geometry and topology II: Closed surfaces, Duke Math. J., 2 (1936), 95-102, Zbl 0013.32201.

[21] J. Myjak and R. Rudnicki, On the typical structure of compact sets, Arch. Math. (Basel), 76(2) (2001), 119-126, MR 1811289 (2002k:46039), Zbl 0981.46018.

[22] Y. Otsu and T. Shioya, The Riemannian structure of Alexandrov spaces, J. Differential Geom., 39 (1994), 629-658, MR 1274133 (95e:53062), Zbl 0808.53061.

[23] G. Perelman, A.D. Alexandrov spaces of curvature bounded below, II, preprint.

[24] G. Perelman, Elements of Morse theory on Aleksandrov spaces, St. Petersburg Math. J., 5 (1994), 205-213, MR MR1220498 (94h:53054), Zbl 0815.53072.

[25] H. Poincaré, Sur les lignes géodésiques des surfaces convexes, Trans. Amer. Math. Soc., 6 (1905), 237-274, JFM 36.0669.01.

[26] A. Rivière, Nervure d'un ouvert d'un espace euclidien, J. Sci. Univ. Tehran Int. Ed., 1 (1996), 1-24, MR 1613382 (99g:53010), Zbl 0614.57009.

[27] K. Shiohama and M. Tanaka, Cut loci and distance spheres on Alexandrov surfaces, Actes de la Table Ronde de Géométrie Différentielle (Luminy, 1992), 531-559, Sémin. Congr., 1, Soc. Math. France, Paris, 1996, MR 1427770 (98a:53062), Zbl 0874.53032.

[28] C. Vîlcu, On degenerate convex surfaces, preprint.

[29] C.K. Yap, An $O(n * \log n)$ algorithm for the Voronoi diagram of a set of simple curve segments, Discrete Comput. Geometry, 2 (1987), 365-393, MR 0911190 (88m:68056), Zbl 0628.68042.

[30] L. Zajíček, Sets of $\sigma$-porosity and sets of $\sigma$-porosity $(q)$, Casopis Pěst. Mat., 101 (1976), 350-359, MR 0457731 (56 \#15935), Zbl 0341.30026.

[31] T. Zamfirescu, Many endpoints and few interior points of geodesics, Invent. Math., 69 (1982), 253-257, MR 0674405 (84h:53088), Zbl 0494.52004.

[32] T. Zamfirescu, Using Baire categories in geometry, Rend. Sem. Mat. Univ. Politec. Torino, 43 (1985), 67-88, MR 0859850 (87j:52007), Zbl 0601.52004.

[33] T. Zamfirescu, How many sets are porous?, Proc. Amer. Math. Soc., 100 (1987), 383-387, MR 0884484 (88c:54029), Zbl 0625.54036.

[34] T. Zamfirescu, The nearest point mapping is single valued nearly everywhere, Arch. Math., 54 (1990), 563-566, MR 1052977 (91k:41061), Zbl 0715.54013.

[35] T. Zamfirescu, Conjugate points on convex surfaces, Mathematika, 38 (1991), 312317, MR 1147829 (93e:52005), Zbl 0759.52005.

[36] T. Zamfirescu, Conjugate points and closed geodesic arcs on convex surfaces, Geom. Dedicata, 62 (1996), 99-105, MR 1400984 (97g:52006), Zbl 0866.53050.

[37] T. Zamfirescu, On some questions about convex surfaces, Math. Nachr., 172 (1995), 313-324, MR 1330637 (96e:52004), Zbl 0833.53004.

[38] T. Zamfirescu, Closed geodesic arcs in Aleksandrov spaces, Rend. Circ. Mat. Palermo (2) Suppl., 50 (1997), 425-430, MR 1603026 (99b:53063), Zbl 0889.53027.

[39] T. Zamfirescu, Extreme points of the distance function on convex surfaces, Trans. Amer. Math. Soc., 350 (1998), 1395-1406, MR 1458314 (98i:52005), Zbl 0896.52006.

[40] T. Zamfirescu, Dense ambiguous loci and residual cut loci, Rend. Circ. Mat. Palermo (2) Suppl., 65(II) (2000), 203-208, MR 1809155 (2001j:52007), Zbl 0982.53034. 
[41] N.V. Zhivkov, Compacta with dense ambiguous loci of metric projections and antiprojections, Proc. Amer. Math. Soc., 123 (1995), 3403-3411, MR 1273531 (96a:41044), Zbl 0842.41024.

Received May 31, 2001 and revised November 7, 2003

FACHBEREICH MATHEMATIK

UNIVERSITÄT DORTMUND

44221 DorTMund

Germany

E-mail address: Tudor.Zamfirescu@math.uni-dortmund.de 